Neurosurg Focus 23 (6):E5, 2007

\title{
Proton radiosurgery in neurosurgery
}

\author{
Clark C. Chen, M.D., Ph.D., ${ }^{1,2}$ Paul Chapman, M.D., ${ }^{1,2}$ Joshua Petit, M.D., 2 \\ AND JAY LOEFFLER, M.D. ${ }^{2}$ \\ Departments of ${ }^{\prime}$ Neurosurgery and ${ }^{2}$ Radiation Oncology, Massachusetts General Hospital, \\ Boston, Massachusetts
}

\begin{abstract}
Object. Photon energy deposition from gamma or photon sources follows the law of exponential decay. Consequently, energy is deposited over the entire path of the radiation beam, resulting in dose distribution before and after the target is reached. In contrast, the physical properties of protons are such that energy deposition occurs with no exit dose beyond the target volume. Therefore, relative to photons, proton beams represent a superior platform for the administration of radiosurgery.

Methods. In this review, the authors will discuss the fundamental principles underlying photon- and proton-based stereotactic radiosurgery (SRS). The clinical efficacy of proton-based SRS in the treatment of arteriovenous malformations, vestibular schwannomas, and pituitary adenomas is reviewed.

Results. Direct comparisons of clinical results attained using photon- and proton-based SRS are confounded by a bias toward reserving proton beams for the treatment of larger and more complex lesions. Despite this bias, the clinical outcomes for proton-based SRS have been excellent and have been at least comparable to those for photonbased treatments.

Conclusions. The physical properties of proton radiation offer superior conformality in dose distribution relative to photon irradiation. This advantage becomes more apparent as the lesion size increases and will probably be magnified with the development of intensity-modulated proton techniques. (DOI: $10.3171 /$ FOC-07/12/E5)
\end{abstract}

\section{KEY WORDS - arteriovenous malformation - photon-based stereotactic radiosurgery - pituitary adenoma $\quad$ proton-based stereotactic radiosurgery $\bullet$ vestibular schwannoma}

$\mathrm{S}$ TEREOTACTIC radiosurgery refers to the delivery of conformal, high-dose radiation to a region of diseased tissue, with relative sparing of the surrounding healthy tissues. As initially proposed by Dr. Lars Leksell, ${ }^{13}$ SRS is achieved by converging multiple, nonparallel radiation beams on a target lesion, thereby minimizing the radiation received in each individual beam path. Since its inception, the development of radiosurgery has largely revolved around the use of photon or gamma delivery systems. This bias is largely due to the relative ease with which photon radiation can be generated and manipulated as compared to protons. Currently, there are 23 clinical proton radiotherapy facilities worldwide, with 4 hospital-based centers available in the US..$^{22}$ Of these US centers, only 2 offer radiosurgery. In contrast, there are $>200$ Gamma

Abbreviations used in this paper: AVM = arteriovenous malformation; $\mathrm{CGE}=$ cobalt gray equivalent; $\mathrm{MGH}=$ Massachusetts General Hospital; RBE = relative biological effect; SRS = stereotactic radiosurgery; STAR = stereotactic alignment for radiosurgery; UCB-LBL = University of California Berkeley, Lawrence Berkeley Laboratory; VS = vestibular schwannoma.
Knife centers worldwide, and many more linear accelerator-based radiosurgery programs.

Despite requiring specialized facilities for its generation and manipulation, proton radiation offers superior dose conformality relative to photon irradiation, and thereby minimizes the morbidities associated with irradiation of normal tissue, including secondary tumor formation. Recognition of this property has fueled growing interest in the development of proton-based SRS. In this article we review the fundamental differences between photon- and protonbased SRS and survey the current literature to review the clinical efficacy of proton-based SRS. Future developments aimed to optimize the use of proton-based SRS are described.

\section{Photon-Based SRS}

In modern physics, photons are defined as an elementary particle that carries electromagnetic radiation of all wavelengths, including gamma rays, x-rays, ultraviolet light, visible light, infrared light, microwaves, and radiowaves. ${ }^{7}$ Of this spectrum of radiation, gamma rays and $\mathrm{x}$-rays are of particular importance in intracranial radiosurgery because 


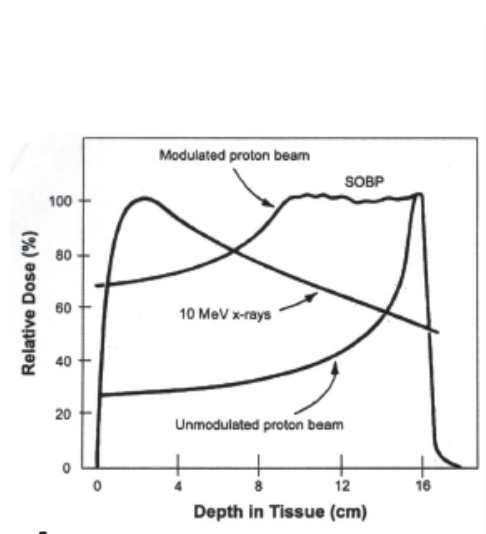

A

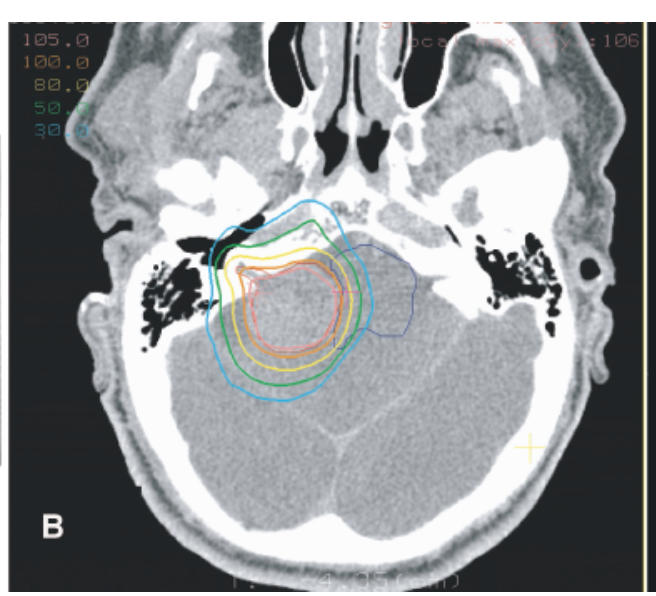

FIG. 1. A: Graph showing a depth-dose curve for an unmodulated proton beam, 10-MeV photon radiation, and spreadout Bragg peak (SOBP) proton radiation. The dose distribution of a proton beam consists of a slowly rising dose, a rapid rise to a maximum (also known as the Bragg peak), followed by a fall to near zero. As a result, a significant portion of the beam energy is deposited in a small volume. In contrast, the energy deposition of photon irradiation is dictated by the laws of exponential decay and is distributed over a larger volume. Because of the sharpness of the Bragg peak, a single monoenergetic proton beam will only irradiate an area approximately the size of a pituitary gland. Irradiation of larger targets is achieved by superimposing proton beams of different energies. The end result of this superposition is known as the spread-out Bragg peak. B and C: Comparative treatment plans are shown for a large VS to be irradiated using proton-based SRS and conformal photon-based SRS. The various isodose lines are as indicated in the upper left corner of each panel. The comparison reveals that proton-based SRS minimizes irradiation of the nontarget tissues relative to photon-based SRS.

they possess sufficient energy to penetrate the cranium and achieve intracranial energy deposition. Gamma rays are produced by subatomic particle interactions, such as radioactive decay of cobalt-60, whereas x-rays are derived from colliding accelerated electrons with a target metal. Despite the differences in the way in which these forms of radiation are produced, their patterns of energy deposition follow the law of exponential decay (Fig. 1A). Consequently, with each individual beam path, substantial radiation to nontarget tissues takes place. Morbidity related to this radiation is minimized by increasing the number of beam paths as well as various methods of beam shaping and intensity modulation. ${ }^{3}$

A plethora of names has been applied to systems designed to deliver gamma or $\mathrm{x}$-irradiation for the purpose of intracranial radiosurgery, including Gamma Knife, CyberKnife, XKnife, and tomotherapy. Regardless of the terminology and mechanical construction, these systems are fundamentally similar in that photon-based radiation is used. To date no significant difference in clinical efficacy has been demonstrated when these various systems are compared. ${ }^{1}$

\section{Proton-Based SRS}

In contrast to photons, protons are generated by stripping an atom of its electron and accelerating the residual proton in the magnetic field of a cyclotron or a synchrocyclotron. ${ }^{12}$ The pattern of energy distribution of a proton beam consists of an entrance region of a slowly rising dose, a rapid rise to a maximum (known as the Bragg peak), and a fall to near zero (Fig. 1A). This property is in fundamental contrast with the exponential decay of photons, in that irradiation of normal tissue within each beam path is minimized. Because of the sharpness of the Bragg peak, a single monoen- ergetic proton beam will only irradiate an area approximately the size of a pituitary gland, ${ }^{16}$ a property that defined its initial clinical application. Irradiation of larger targets is achieved by superimposing proton beams of different energies, thereby generating a dose distribution that provides a moderate entrance dose; a uniform, high dose within the target tissue; and zero dose beyond the target (Fig. 1A). This superposition is known as the spread-out Bragg peak. Most proton treatments are delivered in this manner. When compared with photon-based treatments, proton-based SRS achieves higher conformality to minimize radiation to nontargeted areas (Fig. 1B).

The RBE is a measurement used to compare the biological effects of different types of radiation. It is defined as the ratio of the radiation dose from a reference source required to produce the same biological effect as that of another radiation source. ${ }^{6}$ Historically, cobalt- 60 has been used as the reference radiation source for RBE calculations. For protons, the average RBE reported for proton beams has ranged from 1.1 to 1.2 , with extreme values ranging from 0.7 to $2.1 .^{6,17}$ It is clear that there are insufficient experimental data to predict accurately the RBE of protonbeam radiation for all circumstances. To ensure aggressive treatment, an RBE of 1.1 is semi-arbitrarily selected as the $\mathrm{RBE}$ for protons. The calculated RBE dose is referred to as the CGE. Although the proton RBE of 1.1 was initially semi-arbitrarily selected, subsequent clinical experiences with thousands of treated patients have confirmed its clinical utility. $2,8,14,27$

\section{Advent of the STAR Device and Mobile Beam Gantry for Proton-Based SRS}

Proton-based SRS was pioneered by Dr. Raymond Kjellberg of the MGH at the Harvard Cyclotron Laboratory in 

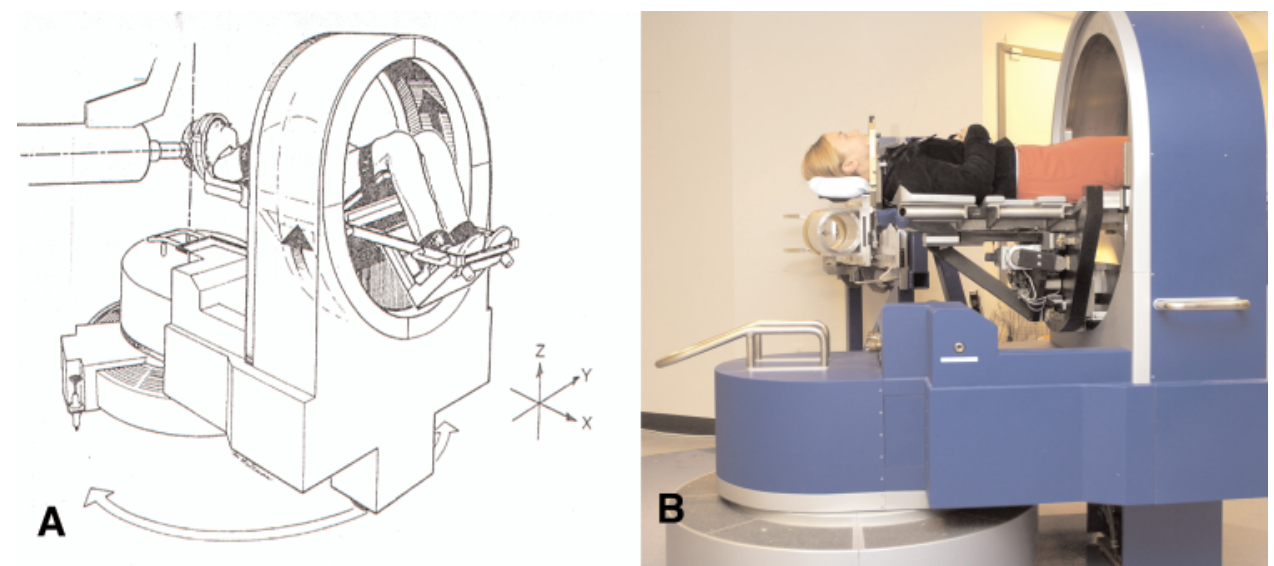

FIG. 2. Drawing (A) and photograph (B) showing the STAR system at the MGH-Northeast Proton Therapy Center. An isocentric patient positioning device designed to rotate the patient around a fixed proton-beam line is shown. The unit has 5 degrees of freedom, 3 of them linear and 2 rotational. Using these axes of movement, the patient is positioned so that the target lesion is at the center of the beam isocenter.

the 1960s..$^{10}$ The treatment sessions were initially time-consuming, in large part due to manual positioning of the patient relative to a fixed horizontal beam. To facilitate proton-based SRS, an automatic patient positioning device was developed under the leadership of Dr. Paul Chapman. This came to be known as the STAR device (Fig. 2). With the STAR device, the patient is placed in an immobilizing head frame attached to a couch apparatus that can be rotated relative to a fixed beam portal. Despite this major advancement, the immobility of the beam source made radiation fields from certain angles unavailable. To address this difficulty, a mobile beam source mounted on a gantry (Fig. 3) was developed.

In general, the steps involved in proton-based SRS are similar to those of photon-based SRS. They include the establishment of a fiducial system for target localization, stereotactic imaging, dosimetric planning, and radiation treatment. Because same-day imaging and treatment is not feasible in proton-based SRS (due to the time required for customization of proton beams), we have adapted distinct fiducial systems for photon- and proton-based SRS. For photon-based SRS, the fiducial system for target localization is based on an axis defined by a rigid head frame. This head frame is placed and removed on the day of radiosurgery. For proton-based SRS, steel beads are implanted into the outer table of the patient's skull in an outpatient procedure. The patient subsequently undergoes imaging and treatment on separate days.

\section{Clinical Efficacy of Proton-Based SRS in Various Types of Lesions}

Direct comparisons of clinical results attained using photon- and proton-based SRS are confounded by multiple clinical variables and the absence of randomized trials. A notable confounding factor is the fact that larger and more complex lesions tend to be treated with proton-beam SRS, whereas smaller lesions tend to be treated with photonbeam SRS. Despite this size bias, the clinical efficacy of proton- and photon-based SRS has been shown to be at least comparable.

In the ensuing sections, the clinical efficacy of proton- based SRS in the treatment of AVMs, VSs, and pituitary adenomas is reviewed.

Arteriovenous Malformations. These lesions represent abnormal communications between vessels of disproportionate and unbalanced hydrodynamic stress. The points of abnormal communication are known as the nidus. The annual risk of hemorrhage from an AVM ranges from 2 to $6 \%$, with an associated fatality rate of $10-20 \% .{ }^{4}$ The goal of AVM treatment with radiosurgery is to eliminate the risk of catastrophic intracranial hemorrhage by irradiation of the AVM nidus. ${ }^{19}$ Much of the current knowledge regarding the efficacy and toxicity associated with proton-beam SRS was derived from series of patients treated at the UCB-LBL and the Harvard Cyclotron Laboratory.

Practitioners at the UCB-LBL began treatment of patients with AVMs by using helium ion radiosurgery as early as $1954 .^{5,23-25}$ The biophysical properties of the helium ion

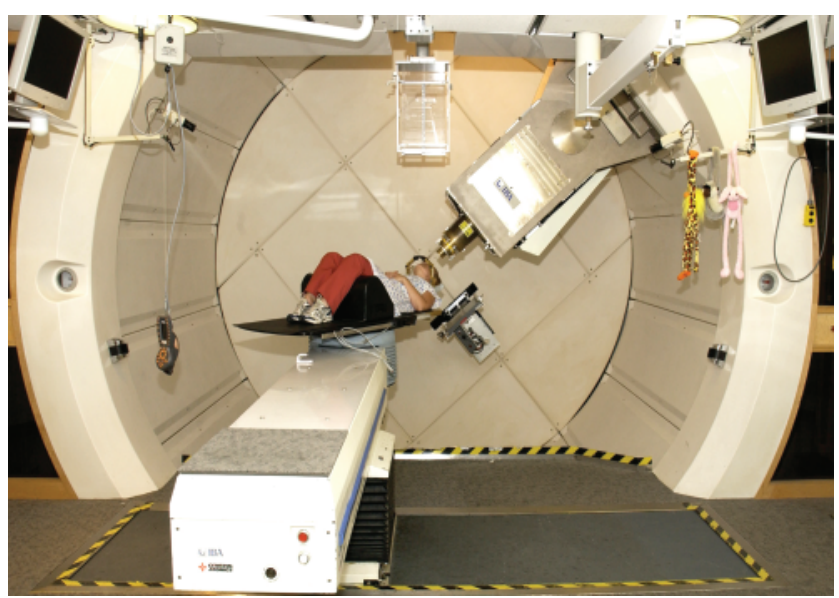

FIG. 3. Photograph showing the proton gantry system at the MGH-Northeast Proton Therapy Center. An isocentric gantry with a mobile proton-beam source is used in conjunction with a robotic patient positioning treatment couch. The robotic unit has 6 degrees of freedom, 3 of them linear and 3 rotational. 

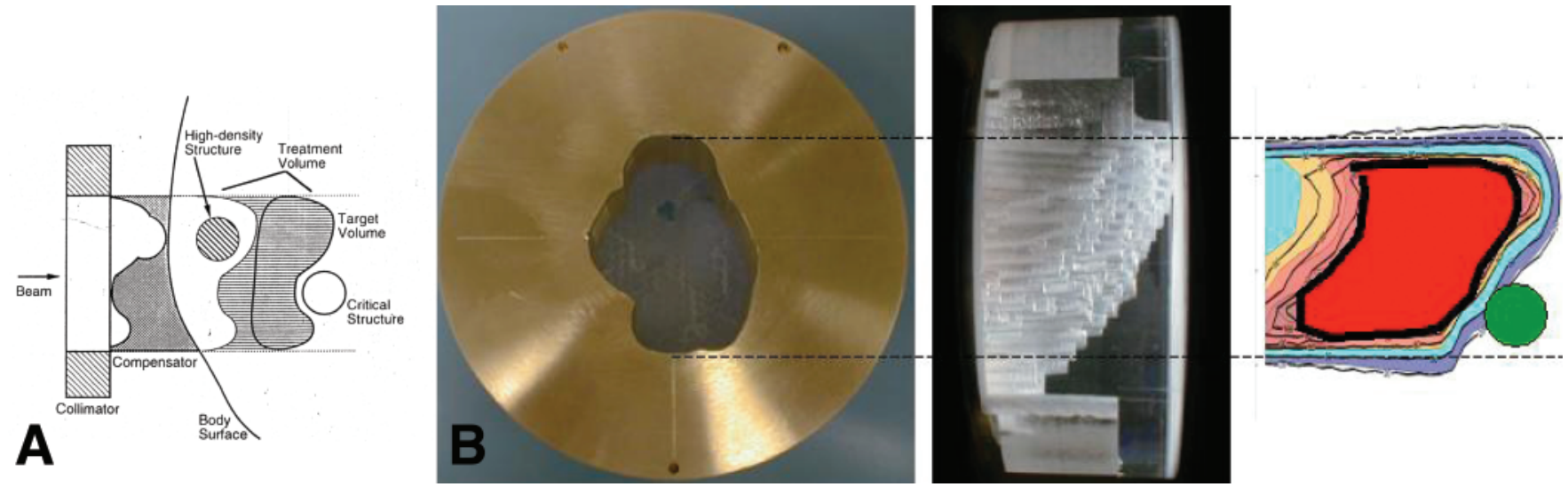

FIG. 4. A: Schema of collimator and compensators. By passing each beam through an aperture shaped to correspond to the cross-sectional area of the lesion in the beam's bird's-eye view, the cross-section of each proton beam is conformed to the corresponding silhouette of the lesion (collimation). By interposing specific materials in the beam's path, energy attenuation is achieved to account for the effects of sloping skin surfaces and tissue nonhomogeneities (compensation). $\mathrm{B}$ : Photographs and drawing of a customized collimator/compensator used in proton-based radiosurgery.

are highly analogous to that of the proton. Therefore, results from these series have been extrapolated to guide the planning of proton-based SRS. In the UCB-LBL experience, doses of 30-45 CGE delivered in single fraction were initially used. At the 3-year follow-up, complete obliteration rates were 100,95 , and $70 \%$ for lesions of $<4$, $4-25$, and $>25 \mathrm{ml}$, respectively. The obliteration rate correlated with the dose delivered and inversely correlated with the size of the AVM. Lesions in the brainstem, thalamus, and basal ganglia were associated with a higher risk of neurological complications.

Although proton-beam SRS has been used to treat cerebral AVMs since 1965, the first report of this use was published by Kjellberg et al. ${ }^{9}$ in 1983. In this series, 75 patients who had undergone proton-beam SRS were followed for $2-16$ years. Four complications were noted among the first 24 patients. After adjustment of the dose prescription, the complication rate was reduced to zero. Sixty-four of the 75 patients had received angiographic follow-up. Of these patients, 20\% showed complete AVM obliteration; 56\% showed AVM size reduction of $>50 \%$; and $13 \%$ had no radiographic changes on follow-up studies. Between 40 and $60 \%$ of all patients treated reported symptomatic improvement. It should be noted that in this series, the AVM sizes were larger on the whole than those reported in later photon-based SRS series. Subsequent proton-based SRS series reported obliteration rates of 67-76\% for AVMs with volumes $<15 \mathrm{ml}$ and $30-40 \%$ for AVMs with volumes $>$ $15 \mathrm{ml}$ at 3-5 years of follow-up. ${ }^{20,21,26}$

Vestibular Schwannomas. These lesions are rare, benign tumors that arise from the Schwann cells associated with the eighth cranial nerve. The goal of VS treatment using radiosurgery is to arrest tumor growth while minimizing the risk of neuropathy in the seventh and eighth cranial nerves. The first report of proton-beam SRS for the treatment of VSs was published by Harsh et al. ${ }^{8}$ and subsequently updated by Weber et al. ${ }^{27}$ Of a total of 88 patients treated using proton-beam SRS, the actuarial local control rate at 2 and 5 years was 95.3 and $93.6 \%$, respectively. The median dose used was 12 CGE prescribed to the $70 \%$ isodose line. The rate of House-Brackmann Grade I/II facial nerve function preservation was $95 \%$. The actuarial hearing preservation rate in those with initial useful hearing was $79.1 \%$ at 2 years, but then it dropped to $21.9 \%$ by 5 years, reflecting characteristic late-effect injury caused by radiation. No facial nerve injury was experienced with target doses of $<17$ CGE. These results are generally comparable to those reported in patients treated using photon-based SRS. ${ }^{15}$

Pituitary Adenomas. There are 2 general categories of pituitary tumors: nonsecreting and secreting lesions. Nonsecreting tumors are usually macroadenomas, defined as being $>1 \mathrm{~cm}$ in diameter. A diagnosis generally results from symptoms associated with growth beyond the sella turcica. Most commonly, patients present with visual field deficits due to optic apparatus compression or neuropathy of the third, fourth, fifth (first division), or sixth cranial nerves from cavernous sinus involvement. Functioning tumors cause an excess secretion of 1 or more pituitary hormones. Depending on the hormone secreted, this results in Cushing disease, acromegaly, hyperprolactinemia, or hyperthyroidism. Secreting tumors are often microadenomas $(<1 \mathrm{~cm}$ in diameter). Except in the case of prolactinomas, surgery is the treatment of choice for both macro- and microadenomas. Radiosurgery is considered in patients with residual or recurrent tumor, or in those who are unlikely to tolerate resection. The goal of treatment is to arrest tumor growth in the case of nonsecreting tumors and to restore endocrine normality in the case of secreting tumors.

An early report of the experience treating acromegaly established the efficacy of proton-based SRS. ${ }^{11}$ In a current update, 22 patients with persistent acromegaly were treated with single-fraction proton-beam SRS (median dose 20 CGE). At a median 6-year follow-up evaluation, 95\% had achieved at least a partial response and 59\% had a complete response, with normalization of insulin-like growth factor-I levels after discontinuation of all medication. ${ }^{18}$ The median time to complete response was 42 months. One third of the patients developed at least 1 new pituitary deficiency, which was corrected with supplementation. Similarly, 38 patients with persistent Cushing disease following surgery were treated with proton-beam SRS (median dose 20 CGE). After a median 5-year follow-up, a complete 
response was achieved in 58\% of patients; the median time to response was 18 months. A new pituitary deficiency occurred in $52 \%$ of patients. Although similar results were reported in the photon-based SRS series, the lesions treated using proton-based SRS were typically larger.

\section{Conclusions}

The physical properties of proton radiation offer superior conformality in dose distribution relative to photon irradiation. This advantage becomes more apparent as the lesion volume increases. The next step in proton radiation is intensity-modulated proton therapy. Current treatments use proton beams that have a uniform intensity across each radiation portal. The radiation is focused onto the target by using custom blocks to shape each portal and custom compensators to control the depth of penetration (Fig. 4). Intensity-modulated proton therapy will enable us to control the actual dose at any given point in each treatment portal by using one of several newly developed techniques. Variable modulation uses a multileaf collimator placed in the head of the machine. These leaves move across the treatment field while the proton beam is activated, blocking a given portion of the radiation field based on the desired intensity at that point. Pencil-beam scanning uses magnets to sweep a small proton beam across the treatment field. By controlling the amount of time the beam spends over any given point, this also allows the delivery of a desired radiation intensity to any point in each treatment field. These techniques will improve the ability to irradiate target tissues selectively while sparing surrounding normal tissues. These improvements will be most substantially realized in the treatment of large, irregularly shaped lesions.

\section{References}

1. Andrews DW, Scott CB, Sperduto PW, Flanders AE, Gaspar LE, Schell MC, et al: Whole brain radiation therapy with or without stereotactic radiosurgery boost for patients with one to three brain metastases: phase III results of the RTOG 9508 randomised trial. Lancet 363:1665-1672, 2004

2. Barker FG II, Butler WE, Lyons S, Cascio E, Ogilvy CS, Loeffler JS, et al: Dose-volume prediction of radiation-related complications after proton beam radiosurgery for cerebral arteriovenous malformations. J Neurosurg 99:254-263, 2003

3. Chen CC, Chapman PH, Loeffler JS: Stereotactic cranial radiosurgery and radiotherapy. UpToDate, 2007 (http: //patients.uptodate.com/topic.asp?file=rad_ther/7736) [Accessed 15 November, 2007]

4. Choi J, Mohr J: Brain arteriovenous malformations in adults. Lancet Neurol 4:299-308, 2005

5. Fabrikant JI, Levy RP, Steinberg GK, Phillips MH, Frankel KA, Lyman JT, et al: Charged-particle radiosurgery for intracranial vascular malformations. Neurosurg Clin N Am 3:99-139, 1992

6. Gerweck L, Kozin S: Relative biological effectiveness of proton beams in clinical therapy. Radiol Oncol 50:135-142, 1999

7. Halliday D, Resnick R, Walker J: Fundamentals of Physics, ed 7. New York: Wiley, 2004, Vol 1

8. Harsh G, Thornton A, Chapman P, Bussiere M, Rabinov J, Loeffler J: Proton beam stereotactic radiosurgery of vestibular schwannomas. Int J Radiat Oncol Biol Phys 54:35-44, 2002

9. Kjellberg RN, Hanamura T, Davis KR, Lyons SL, Adams RD: Bragg-peak proton-beam therapy for arteriovenous malformations of the brain. N Engl J Med 309:269-274, 1983
10. Kjellberg RN, Koehler AM, Preston WM, Sweet WH: Intracranial lesions made by a proton beam, in Haley T, R S (eds): Response of the Nervous System to Ionizing Radiation. Boston: Little, Brown, 1964, pp 3-18

11. Kjellberg RN, Shintani A, Frantz AG, Kliman B: Proton-beam therapy in acromegaly. N Engl J Med 278:689-695, 1968

12. Lawrence E, Edlefsen N: On the production of high speed protons. Science 72:376, 1930

13. Leksell L: The stereotaxic method and radiosurgery of the brain. Acta Chir Scand 102:316-319, 1951

14. Loeffler JS, Smith AR, Suit HD: The potential role of proton beams in radiation oncology. Semin Oncol 24:686-695, 1997

15. Lunsford LD, Niranjan A, Flickinger JC, Maitz A, Kondziolka D: Radiosurgery of vestibular schwannomas: summary of experience in 829 cases. J Neurosurg 102 Suppl:195-199, 2005

16. Miller DW: A review of proton beam radiation therapy. Med Phys 22:1943-1954, 1995

17. Paganetti H, Niemierko A, Ancukiewicz M, Gerweck L, Goitein M, Loeffler J, et al: Relative biological effectiveness (RBE) values for proton beam therapy. Int J Radiat Oncol Biol Phys 53: 407-421, 2002

18. Petit JH, Biller BMK, Swearingen B, Coen J, Ankuciewicz M, Klibanski A, et al: Proton stereotactic radiosurgery is effective and safe in the management of persistent acromegaly. Endocrine Society Abstract P3-785, 2006

19. Pollock B, Lunsford L, Kondziolka D, Maitz A, Flickinger J: Patient outcomes after stereotactic radiosurgery for "operable" arteriovenous malformations. Neurosurgery 35:1-8, 1994

20. Seifert V, Stolke D, Mehdorn HM, Hoffmann B: Clinical and radiological evaluation of long-term results of stereotactic proton beam radiosurgery in patients with cerebral arteriovenous malformations. J Neurosurg 81:683-689, 1994

21. Silander H, Pellettieri L, Enblad P, Montelius A, Grusell E, Vallhagen-Dahlgren $\mathrm{C}$, et al: Fractionated, stereotactic proton beam treatment of cerebral arteriovenous malformations. Acta Neurol Scand 109:85-90, 2004

22. Sisterson J: Particle Therapy Co-Operative Group (PTCOG). Particles Newsletter 35, 2005

23. Steinberg GK, Fabrikant JI, Marks MP, Levy RP, Frankel KA, Phillips MH, et al: Stereotactic heavy-charged-particle Braggpeak radiation for intracranial arteriovenous malformations. $\mathbf{N}$ Engl J Med 323:96-101, 1990

24. Steinberg GK, Fabrikant JI, Marks MP, Levy RP, Frankel KA, Phillips MH, et al: Stereotactic helium ion Bragg peak radiosurgery for intracranial arteriovenous malformations. Detailed clinical and neuroradiologic outcome. Stereotact Funct Neurosurg 57:36-49, 1991

25. Steinberg GK, Levy RP, Fabrikant JI, Frankel KA, Phillips MH, Marks MP: Stereotactic helium ion Bragg peak radiosurgery for angiographically occult intracranial vascular malformations. Stereotact Funct Neurosurg 57:64-71, 1991

26. Vernimmen FJ, Slabbert JP, Wilson JA, Fredericks S, Melvill R: Stereotactic proton beam therapy for intracranial arteriovenous malformations. Int J Radiat Oncol Biol Phys 62:44-52, 2005

27. Weber DC, Chan AW, Bussiere MR, Harsh GR IV, Ancukiewicz M, Barker FG II, et al: Proton beam radiosurgery for vestibular schwannoma: tumor control and cranial nerve toxicity. Neurosurgery 53:577-588, 2003

\footnotetext{
Manuscript submitted September 20, 2007.

Accepted October 5, 2007.

Address correspondence to: Clark C. Chen, M.D., Ph.D., Department of Neurosurgery, 55 Fruit Street, Boston, Massachusetts 02114. email: cchen1@partners.org.
} 